\title{
The contribution of visual persistence to the perceived duration of brief targets
}

\author{
GERALD M. LONG and ROBERT J. BEATON \\ Villanova University, Villanova, Pennsylvania 19085
}

\begin{abstract}
Two experiments were conducted to examine the role of sensory persistence on tasks of perceived duration employing very brief visual stimuli. Using a standard temporal judgment task, the first experiment replicated both the "size effect" and "empty-filled" illusion reported by previous investigators. However, entirely comparable results were also found with a probematching task, which theoretically assesses the degree of persistence exhibited by a stimulus. The second experiment examined the effect of target luminance on perceived duration. Consistent with a sensory persistence interpretation, judgments of duration increased with increasing luminance. The results from the two experiments were discussed in terms of varying degrees of retinal persistence produced by different stimuli. This view was contrasted with currently dominant interpretations that postulate changes in perceived duration to reflect different information-processing requirements across stimulus conditions.
\end{abstract}

Extensive research has focused on the perceived duration of very brief visual stimuli and the numerous subject and task variables affecting such perceptions (cf. Allan, 1979; Thomas \& Weaver, 1975). The reason for much of the interest in the phenomenal duration of such very brief targets rests in the basic working assumption among investigators in the field that significant insight into the processing mechanisms of the visual system may be gained by the determination of variables that influence these perceptions. As Avant, Lyman, and Antes (1975) have suggested, "the differences in apparent duration of tachistoscopic presentations may index the operations of perceptual and preperceptual processing" (p. 253). Variables that can be shown to influence the phenomenal duration of a target are then examined in terms of the type of visual process(es) that might be involved. Hence, it is frequently not the perception of time per se that is of interest in this work, but rather the fact that changes in the perceived duration of very brief flashes may indicate how the visual system extracts and processes information from a tachistoscopic presentation (cf. Thomas \& Cantor, 1976; Thomas \& Weaver, 1975).

In her recent review of the time-perception literature, Allan (1979) has described several different models that have been developed in this area. In general, these conceptualizations emphasize some type of

Requests for reprints should be sent to Gerald M. Long, Department of Psychology, Villanova University, Villanova, Pennsylvania 19085 . The results reported in this study were presented at the meeting of the Eastern Psychological Association, Hartford, Connecticut, April 1980. We wish to thank J. Porter Tuck for his helpful comments concerning the data analysis. stage analysis of the information contained in a brief presentation. Changes in perceived duration under various empirical manipulations are then interpreted in terms of alterations in the nature or degree of processing by one or more of the stages postulated to underlie observers' reports. In one of the best known of current theories, Thomas and his colleagues (Cantor \& Thomas, 1976, 1977; Thomas \& Brown, 1974; Thomas \& Cantor, 1975, 1976, 1978; Thomas \& Weaver, 1975) have proposed two independent, parallel-processing stages that mediate observers' duration judgments. The "ff-processor," or timer, is directly sensitive to the physical duration of the stimulus. The "g-processor," or information processor, is involved with encoding the nontemporal information content of the stimulus. This information can influence the perceived duration of a stimulus because of the time spent processing information, which can vary depending upon both stimulus and instructional conditions. The observers' subjective experience of target duration is then a weighted combination of the output from these two hypothetical processors. Related models in the time-perception literature have proposed specific operations that theoretically mediate performance on duration discrimination tasks (e.g., Allan \& Kristofferson, 1974b; Allan, Kristofferson, \& Wiens, 1971; Kristofferson, 1977), as contrasted with the perceived duration tasks described above. Other investigators have examined the nature of the functional relationships between various stimulus variables and performance on temporal judgment tasks (e.g., Allan \& Kristofferson, 1974a; Eisler, 1976). These psychophysical models have traditionally been concerned with quantifying the proposed operations of the 
internal "timekeeper" whose functioning is assessed by the various temporal discrimination tasks (Allan \& Kristofferson, 1974b).

It is the concern of the present study that much of current theory and research in the perceivedduration literature may have overlooked the critical importance of sensory persistence effects in at least some of the empirical findings. Although some researchers in this field have noted that visual persistence may play a role in their tasks, either its contribution to task performance has been considered very minor (Avant et al., 1975; Craig, 1973) or the persistence is treated as having a rather invariant effect upon performance (Cantor \& Thomas, 1976). We contend that many of the manipulations tested by researchers may directly alter the degree of phenomenal persistence (iconic duration) and in this way affect performance on perceived-duration tasks at a very early, peripheral stage in the visual system. It has long been known (cf. Allen, 1926; Sperling, $1960)$ that the visual system is somewhat sluggish in its functioning so that a brief or rapidly moving stimulus can phenomenally persist beyond its physical duration. Within the last 20 years, much of the research into this persistence has been undertaken within the context of so-called "iconic memory" or shortterm visual storage. Following Sperling's (1960) ground-breaking research, hundreds of studies have focused upon the nature and function of the rapidly decaying trace of a tachistoscopic stimulus that outlasts the physical offset of the stimulus even by hundreds of milliseconds and that can be directly utilized by observers (cf. Coltheart, 1975, 1980; Dick, 1974; Long, 1979b; Sakitt, 1976). Moreover, much of the research within the last few years has indicated that the bulk of the "memory" or storage reflected in these studies may have its basis in simple retinal inertia, that is, the retinal response outlasting the physical stimulus (e.g., Long, 1979a, 1979b; Long, in press; Long \& Sakitt, 1980a, 1980b; Sakitt, 1975, 1976; Sakitt \& Long, 1978, 1979a, 1979b). In other words, contrary to the traditional interpretation of iconic memory, it appears that the phenominal reports by observers as well as the empirical demonstrations of a fading image of a brief stimulus result from a retinal persistence effect.

Given the strong evidence that a brief stimulus persists beyond its physical duration due iargely to peripheral factors (i.e., retinal icons), the present research has sought to demonstrate the contribution of this persistence to several of the phenomena in the perceived-duration literature. It will be shown that at least some of these phenomena can be predicted from a retinal persistence interpretation, and that the cognitive processing models that are currently popular in the time-perception literature may have postulated unnecessariily complex, higher order mechanisms to account for these very same phenomena. Specifically, the present research examines the effects of target size, target luminance, target duration, and target contrast on both a standard perceived-duration task and a common persistence task. It will be argued that the comparability of these specific target manipulations across tasks is treated most parsimoniously in terms of the single process of retinal persistence. Rather than purported demonstrations of the effects of the nontemporal information in a brief stimulus on central processing stages, these results are discussed in terms of basic stimulus parameters that directly influence the clarity and duration of an energy-dependent retinal persistence.

\section{EXPERIMENT 1}

This first experiment is specifically concerned with the "size effect" on duration judgments reported in several previous studies (e.g., Cantor \& Thomas, 1976; Mo \& Michalski, 1972; Thomas \& Cantor, 1975, 1976). The finding of increased duration estimates with increasing target size has been interpreted as evidence for the significant effect of nontemporal information on temporal judgments. As noted previously, to account for effects such as these, Thomas and his colleagues (e.g., Thomas \& Cantor, 1976) have proposed the existence of an information processor that extracts the nontemporal information in a stimulus. It is assumed that larger targets require more (i.e., longer) processing by this mechanism than do smaller targets. The observer's experience of the larger target's duration incorporates this extra "timeto-process" along with the output from the independent timer mechanism, which is directly dependent on the temporal information (i.e., duration) in the target.

An alternate and much more basic interpretation of these findings is suggested from the persistence literature. The experimental conditions employed in the research cited above are appropriate for a strong contribution to performance from sensory persistence effects. Sluggish retinal functioning would result in a gradual fading of the brief targets after their physical offset. Longer flashes contain more energy than brief flashes, and a persistence based upon an energydependent retinal phenomenon would correspondingly increase with increasing duration-at least within the limits of temporal summation exhibited by the retina (Long, 1979a; Long \& Sakitt, 1980a, 1980b). Moreover, the fading image of a large target would be detectable for a longer period than that of a small target. This point has been raised previously by Sakitt and Long (1978), who argue that a photoreceptor interpretation of the locus of iconic storage would predict larger targets to produce phenomenally longer icons. They propose that, reflecting the wellknown tradeoff between luminance and acuity, an 
icon of a large target will still be discriminable when the icon from a small target is not. Their experimental findings on an iconic memory task supported this interpretation.

Experiment 1 investigated the effect of target size on two tasks: a temporal judgment task from the current time-perception literature and a standard persistence task from the iconic-memory literature. A demonstration of a size effect on the latter persistence task, which uses the identical stimuli and viewing conditions as the former task, would support the interpretation that the similar size effect on the perceived-duration task results from discriminable persistence differences across stimuli of varying size.

A closely related observation in the duration literature was also examined in this first experiment, because it, too, is believed to result from the same underlying process of retinal persistence. In the context of perceived duration, the so-called "emptyfilled" illusion refers to the fact that an empty (blank) interval containing no other stimuli is perceived as briefer than a filled interval of the same physical duration that contains a high-contrast target (e.g., Thomas \& Cantor, 1975; Thomas \& Weaver, 1975). In the Thomas and Cantor model described above, this finding is interpreted as reflecting the different amount of contribution in the two cases from the information-processing mechanism. The perceived duration of the blank field results from the output of the timer mechanism alone without any contribution from the information processor, because the field contains no nontemporal information to be processed. The filled interval, by definition, is filled with some information, and the information processor is then engaged by the duration task. The alternate explanation raised here concerns the continuum of discriminable persistence that is believed to result from targets of varying size. The fading icon of a large target is still detectable at longer intervals following target offset than is that of a small target. If the size of the target stimulus is reduced to the extreme, it falls below detection threshold, and the stimulus becomes a blank field. With a blank field as the target, no high-contrast target is present in the field, and minimal persistence would then be expected. Hence, the single variable of clarity of the resulting icon may be sufficient to account for this "illusion" -along with the "size effect" noted above.

\section{Method}

Subjects. Fifteen subjects (10 male, 5 female) participated in Experiment 1. These subjects were undergraduates at Villanova University who participated in the research for partial completion of a course requirement in general psychology. Each subject participated in a single experimental session lasting between 60 and $90 \mathrm{~min}$. If subjects normally wore spectacles, they also wore them during the experimental session.

Apparatus and Stimuli. A Scientific Prototype 3-channel tachistoscope (Model 320-GB) was employed for the presentation of all stimuli and background fields. For one of the conditions described below, a brief auditory tone was required. A 2,000- $\mathrm{Hz}$ tone was provided by a Western Electric Oscillator (19C) and a Bogen Amplifier (CHB 35A). The constant duration of the tone $(150 \mathrm{msec})$, the variable interval between the visual target and the tone, and the constant interval between the auditory warning signal ("click" of toggle switch) and the presentation of the visual target ( $500 \mathrm{msec}$ ) were controlled by three Hunter Timers (111-C) that interfaced with the tachistoscope. The luminances of the target and background fields are described below. All targets were high-contrast $(95 \%)$ black dots of variable diameter, positioned in the center of the $8.3 \times 5.9 \mathrm{deg}$ white stimulus card. Pre- and poststimulus fields were of the same white cards as the stimulus fields. A small black fixation point was located in the center of these background fields.

Procedure: Size of stimulus effects. Two different tasks were employed in the investigation of target size effects. The first task was a standard perceived-duration task used in several studies by Thomas and Cantor (e.g., 1975, 1976). On each trial, the observers were instructed to report whether their subjective experience of the target presentation was "short," "medium," or "long." Actual target duration was either 40 or $70 \mathrm{msec}$, and the three size targets were employed. These targets were black dots on white backgrounds and were .7, .9, and $1.1 \mathrm{deg}$ in diameter. (This is the same range of values employed in Thomas \& Cantor, 1975.) These dots were centered on the white backgrounds and were superimposed over the position occupied by the small fixation point on the pre- and postexposure fields. The luminance of the white areas of the target and background fields was held constant at $25 \mathrm{fL}\left(85.6 \mathrm{~cd} / \mathrm{m}^{2}\right)$.

Before the experimental trials were begun, each observer received 5-10 min of practice in which he was shown all six possible combinations of target size and target duration. The purpose of the brief practice was to familiarize the subjects with the range of impressions of target duration they would be experiencing during the actual experiment so they could "anchor" their judgments of "short," "medium," and "long" from the very beginning of the trials. They were not told that the presentations varied in duration between 40 and $70 \mathrm{msec}$.

Following the practice, each of five observers received 120 total trials, which consisted of 20 presentations of the six size and duration conditions that were randomized throughout the 120 total trials with the constraint that each condition occur within every six trials. Following each presentation, the subject verbally reported whether the phenomenal impression of the target on that trial was "short," "medium," or "long." The experimenter recorded these responses and proceeded to the next trial without giving the subjects any feedback about their responses. After 60 trials, the subjects were allowed a brief 2-3-min rest period.

The other task employed in this first experiment was a probematching task frequently used in the persistence literature. Basically, observers were required to adjust a $150-\mathrm{msec}$ tone $(2,000 \mathrm{~Hz})$ so that it coincided with the end of any impression from the visual target. It is important with this procedure that observers be instructed to attend to the fading trace (i.e., persistence) of the target rather than to the offset of the target. Sakitt and Long (1979a) have shown that observers can easily make this distinction and that the relationship between performance and stimulus parameters (e.g., luminance, duration, wavelength) is quite different under the two sets of instructions. As several researchers have found, probe-to-offset instructions result in inverse energy effects (e.g., Bowen, Pola, Matin, 1974; Efron, 1970; see Coltheart, 1980 , for a recent review of these studies). However, probe-topersistence instructions result in positive energy effects and would appear to assess more directly the impressions of a fading stimulus trace reported by observers on standard iconic memory tasks (cf. Long, 1979a, in press; Sakitt \& Long, 1979a). Because of this critical distinction, observers were specifically instructed to attend to the fading trace of the target.

The identical target conditions employed in the perceived- 
duration task described above were used on this persistence task. On each trial, the experimenter initially adjusted the interval for the probe so that the probe either clearly preceded or clearly followed the end of the target persistence. If the observer responded that the probe was "early," the experimenter then increased the interval by $50 \mathrm{msec}$ (ascending series); if the observer responded that the probe was "late," the experimenter then decreased the interval by $50 \mathrm{msec}$ (descending series). This procedure was repeated until the observer reported the end of the persistence and the onset of the probe to "match." The experimenter recorded this value, selected the appropriate size and duration conditions for the next trial, and repeated the above procedure. As with the perceived-duration task, all subjects first received 5-10 min of practice to familiarize them with the task.

Because this probe-matching task is relatively more timeconsuming than the temporal judgment task of Thomas and Cantor, a between-subjects design was employed for the variable of target duration. Five subjects were run with a constant target duration of $40 \mathrm{msec}$ and the three target sizes, and five other subjects were run with a constant target duration of $70 \mathrm{msec}$ and the same three target sizes. Each target size was run in pairs of trials: one ascending adjustment series and one descending adjustment series. Then, a second target size was employed for one ascending trial set and one descending trial set, and the third target size was then examined in the same manner. The order of the three target sizes was randomized over the 30 trial pairs constituting the 60-90-min experimental session. Each target size was therefore presented to each observer on 20 trials, half of which employed a series of ascending adjustment presentations and half of which employed descending adjustment presentations. On each trial, the experimenter varied the starting ISI value from that used in the previous series of the same type. After 30 trials, a brief 2-3-min rest period was allowed before the second half of the session was begun.

Procedure: Empty-filled illusion. Five additional subjects were run with the probe-matching task in an attempt to determine the relative degree of persistence from an empty field and from the same field containing a high contrast stimulus (.9-deg black dot). Two target durations were used $(40$ and $70 \mathrm{msec})$ so that each observer received four possible stimulus conditions. The luminances of the white backgrounds were again constant at $25 \mathrm{fL}$.

Each of the four stimulus conditions was presented in 20 trials that were run in 10 pairs of an ascending and descending adjustment series. The order of the four possible types of pairs was randomized uniquely for each observer. As described above, within each trial, the observer simply reported whether the probe was "early" or "late" with respect to the end of the persistence from the target. If the observer responded "early" ("late"), the experimenter increased (decreased) the ISI for the probe in 50 -msec steps until the observer reported "match." This value was recorded by the experimenter as an estimate of the degree of persistence for that target, and the experimenter then adjusted the apparatus for the next trial of either ascending or descending presentations. The observer was given a brief rest after 40 pairs of trials. Before the experimental trials were begun, each observer received 5-10 min of practice on matching the probe to the end of a target's persistence.

\section{Results and Discussion}

The temporal judgments of "short," "medium," and "long" on the perceived-duration task were coded 0,1 , and 2 , respectively, following the analysis employed by Thomas and Cantor $(1975,1976)$. For each of the six stimulus conditions (two durations, three sizes), the mean responses for the five observers were then determined. These data, averaged across subjects, are presented in Figure 1. For both the 40-

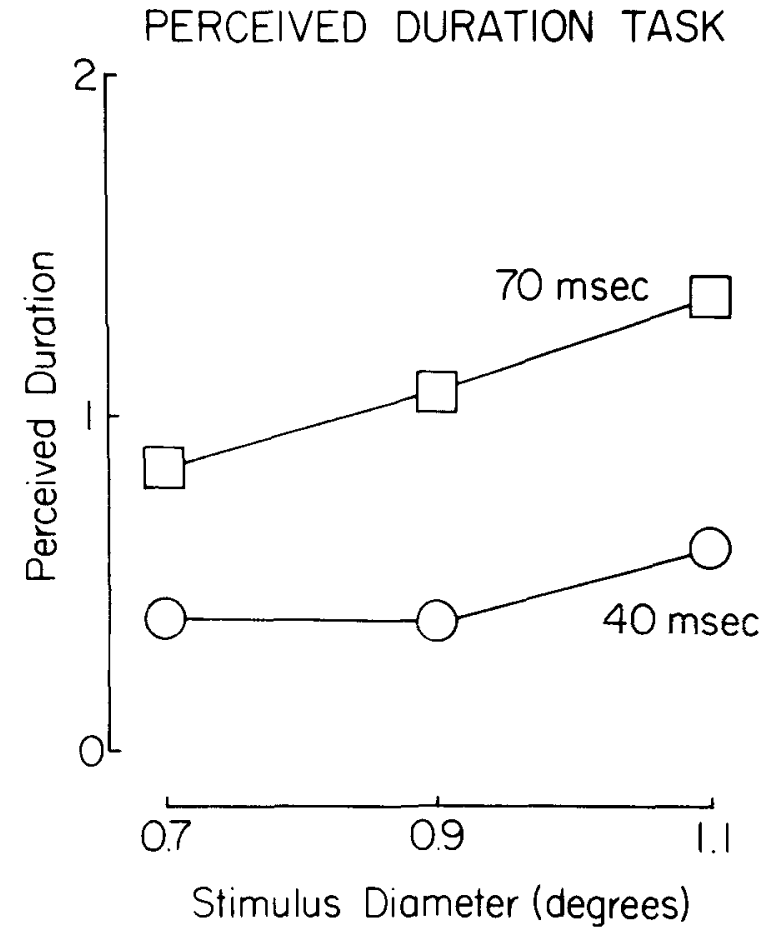

Figure 1. Temporal judgments on the perceived-duration task, averaged over observers, for the two target durations and three target sizes (Experiment 1).

and 70-msec targets, there is a positive effect of increasing target size, although, as Thomas and Cantor (1975) also found, this appears somewhat more pronounced for the longer duration condition. The main effect of target size is significant $[F(2,8)=$ $6.6, p=.02]$, and the other main effect of target duration is also significant $[F(1,4)=24.8, p<.01]$. The interaction of target duration and target size fails to reach significance $[F(2,8)=3.4, p>.05]$. This pattern of results of the perceived-duration task in the present study replicates findings of target duration and target size reported in previous research.

Figure 2 presents the findings for the two groups of observers run under the identical stimulus conditions as those in Figure 1, but with the probe-matching task. The obtained estimates of persistence (latency for probe) reveal the very same effects of target duration and target size as obtained on the perceivedduration task in Figure 1. The effect of target size on probe latency is highly significant $[F(2,16)=19.9$, $p<.001]$, as is the effect of target duration $[F(1,8)=$ $9.3, \mathrm{p}<.02] ;$ the interaction fails to reach significance $[F(2,16)=2.5, p>.10]$. These results are consistent with those reported on a different iconic persistence task with other stimulus conditions (Sakitt \& Long, 1978).

The comparability of results presented in Figures 1 and 2 , while not by any means proving that the same processes underlie the two tasks, is consistent 


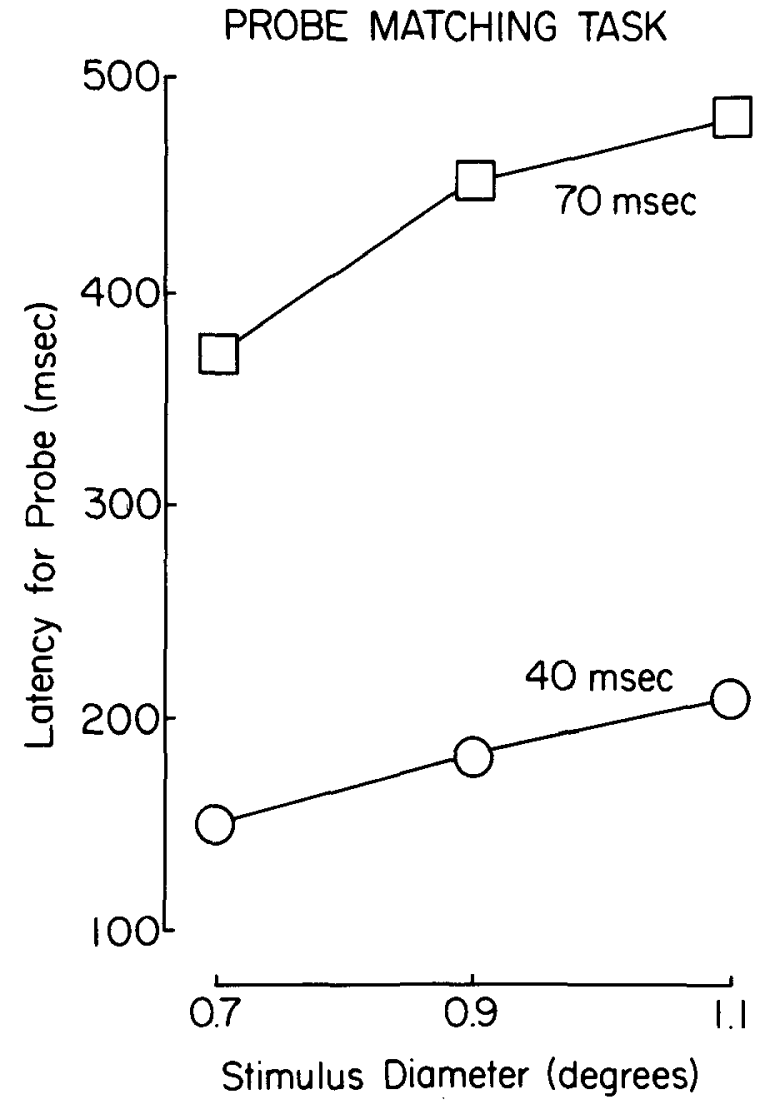

Figure 2. Adjusted latencies for the auditory probe on the probe-matching task, averaged over observers, for the two target durations and three target sizes (Experiment 1).

with the claim raised here that the same sensory process mediates performance on the tasks represented in the two figures. The authors favor the interpretation that this process is simple retinal persistence. It is proposed that the subjective duration of a brief target is strongly influenced by the sensory persistence that follows its physical offset, and that this persistence differs across such stimulus conditions as target size. As indicated previously, this conceptualization is consistent with current persistence literature and exhibits far greater parsimony than the hypothetical construct that has been alternately proposed of an "information processor" taking sequential samples of information from a brief target.

The findings from the investigation of the effect of the empty-filled illusion on persistence estimates (probe-matching task) present further support for this interpretation. Figure 3 depicts the mean latency for the probe as a function of type of target for both the 40- and 70-msec durations. As predicted, the effects of both target duration $[F(1,4)=54.0, p=.002]$ and type of stimulus $[\mathrm{F}(1,4)=44.0, \mathrm{p}=.003]$ are highly significant. The interaction is also significant $[F(1,4)$ $=16.7, p=.015]$, which appears to result from the fact that increasing target duration has a somewhat

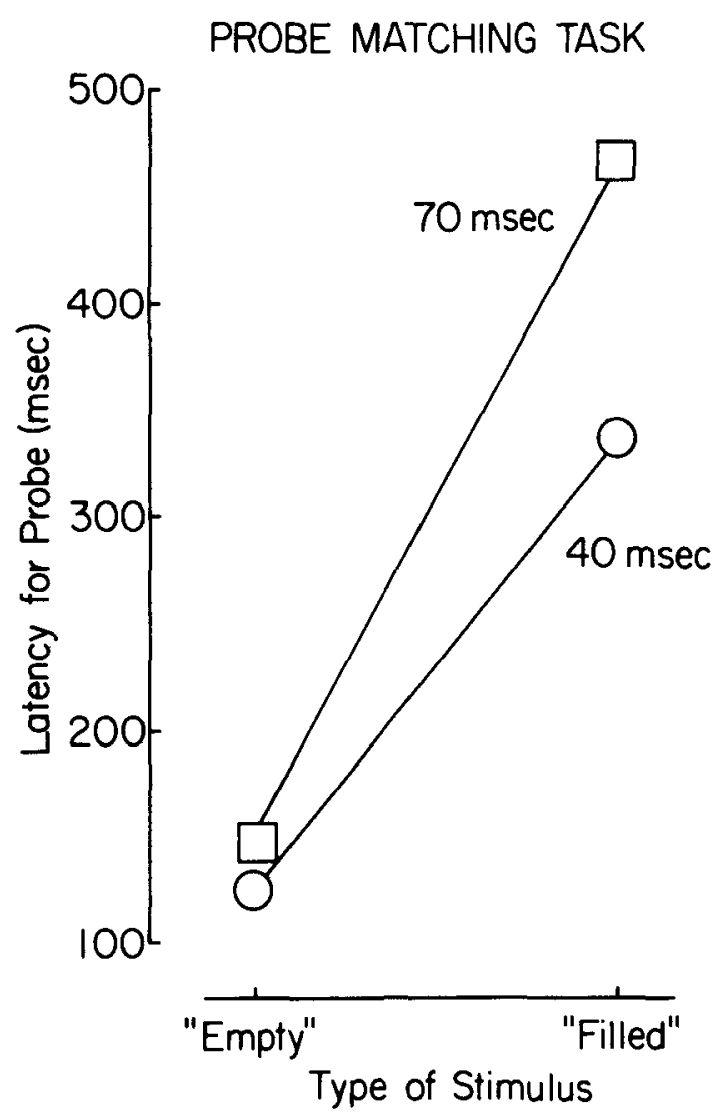

Figure 3. Adjusted latencies for the auditory probe on the probe-matching task, averaged over observers, for the two target durations and two types of target (Experiment 1).

greater effect on the resulting persistence for the highcontrast target. This finding is not inconsistent with the argument proposed here. The relationship between phenomenal persistence and target duration may well be expected to differ for the different types of targets. Because empty-field targets produce the predicted minimal persistence (with bright pre- and postexposure fields), increasing the duration of such targets will have a much less significant effect on phenomenal duration than the similar manipulation for high-contrast targets, which produce appreciable persistence.

\section{EXPERIMENT 2}

The results from the first experiment are entirely consistent with the proposal outlined in the introduction that at least some of the phenomena in the perceived-duration literature may be due to peripheral (sensory) rather than central (cognitive) variables. The single assumption of a fading trace of a target resulting from retinal persistence was shown to be able to account for the "size effect" and "emptyfilled" illusion in the temporal literature involving brief visual stimuli. This second experiment addresses 
a common assumption in that same literature that is directly at odds with the retinal persistence interpretation favored for Experiment 1 . Specifically, it has been claimed by some investigators that the phenomenal duration of a brief target is independent of the intensity of those targets (e.g., Thomas \& Cantor, 1975; Thomas \& Weaver, 1975). This claim is not consistent with the retinal persistence explanation put forth in Experiment 1. Numerous studies in the iconic memory literature have demonstrated that visual persistence increases with increasing target luminance (e.g., Keele \& Chase, 1967; Long \& Sakitt, 1980a, 1980b; Sakitt, 1975, 1976; Sakitt \& Long, 1978, 1979a, 1979b). However, although this result in the persistence literature would appear to contradict the proposal made in the first experiment, the discrepancy may in fact be more apparent than real. The study repeatedly cited as demonstrating the independence of duration judgments and target luminance is that of Allan, Kristofferson, and Wiens (1971). In that study, the luminance of a $120-\mathrm{msec}$ target light was varied over the specific values of 11,13 , and $15 \mathrm{fL}$, and no effect of varying luminance level on that target's discriminability from a $100-\mathrm{msec}$ light of constant $15-\mathrm{fL}$ luminance was obtained. However, it is questionable to assume that "total energy of the field does not affect perceived duration"' (Thomas \& Weaver, 1975, p. 366) based on a total target range in the Allan et al. study of only $4 \mathrm{fL}$. Direct support for this caution can be found in the persistence literature. Some of the early work in iconic memory also reported limited luminance effects based on similar restricted observations (e.g., Eriksen \& Collins, 1968), but later work that varied luminance over a greater range has consistently found highly significant effects of luminance on persistence (cf. Long, in press; Sakitt, 1976). In light of the critical importance of the range of luminance employed, in this second experiment the effects of luminance on the Thomas and Cantor perceived-duration task will be examined over a much greater range of values than that employed by Allan et al. (1971). If the retinal persistence interpretation of performance on that task is correct, greater estimates of perceived target duration are predicted for the higher luminance targets on this temporal judgment task.

\section{Method}

Subjects. Five subjects (two male, three female) were employed in Experiment 2. These subjects participated in the research as a means of fulfilling a course requirement in general psychology at Villanova University. Each subject participated in a single session lasting between 60 and $90 \mathrm{~min}$.

Apparatus and Stimuli. The same basic apparatus described in Experiment 1 was again employed. The stimulus was a constantsize black dot $(.9 \mathrm{deg})$ centered on a white background subtending $8.3 \mathrm{deg}$ horizontal $\times 5.9 \mathrm{deg}$ vertical. Target duration was either 40 or $70 \mathrm{msec}$. The major difference from the first experiment concerns the luminance levels employed. The luminance of the white background was varied over three levels: $5.0,15.0$, and $30.0 \mathrm{fL}$ (i.e., $17.1,51.4$, and $102.8 \mathrm{~cd} / \mathrm{m}^{2}$ ). The luminances of the preand postexposure fields were held constant at $15 \mathrm{fL}$.

Procedure. The perceived-duration task described in Experiment 1 was employed. The observer was instructed to respond "short," "medium," or "long" after each presentation. Following a brief series of practice trials in which the observers were familiarized with the six possible stimulus presentations (two durations, three luminance levels), a total of 120 experimental trials were administered. The order of six conditions ( 20 trials each) was randomized over the 120, with the single constraint that each condition occur within every six trials. After 60 trials, a brief 2-3-min rest period was allowed before the last half of the experimental session was begun.

\section{Results and Discussion}

The results for Experiment 2, averaged across the five observers, are shown in Figure 4. As in Experiment 1, the "short," "medium," and "long" judgments on the perceived-duration task were coded 0 , 1 , and 2 , respectively, and then analyzed with a traditional analysis of variance. Consistent with the results in the first experiment, the main effect of target duration is highly significant, with the 70-msec target judged longer than the 40-msec target $[F(1,4)=$ $18.1, \mathrm{p}<.02]$. Of particular interest in this experiment is the significant main effect of target luminance, indicating that as target luminance is increased, phenomenal duration also increases $[\mathrm{F}(2,8)=196.5$, $\mathrm{p}<.0001]$. This indicates that perceived duration can be seriously affected by altering the luminance

\section{PERCEIVED DURATION TASK}

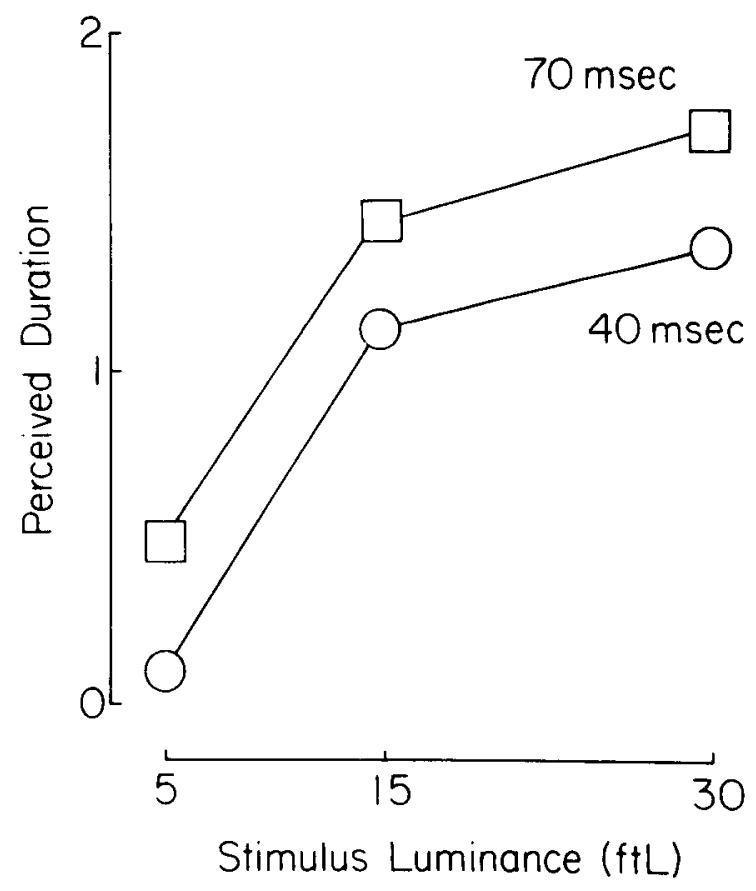

Figure 4. Temporal judgments on the perceived-duration task, averaged over observers, for the two target durations and three target luminances (Experiment 2). 
levels of the targets. Consider, for example, the relative scores for just two conditions: the $70-\mathrm{msec} /$ 5-fL target and the 40-msec/15-fL target (see Figure 4). The latter, briefer condition is judged significantly longer than the former condition $[t(4)=4.51, p=.011]$. This comparison across durations is even more pronounced if the more intense $40-\mathrm{msec} / 30-\mathrm{fL}$ condition is compared with the same $70-\mathrm{msec} / 5-\mathrm{fL}$ target $[t(4)=6.44, p=.003]$. Hence, a more intense but brief target may be judged phenomenally longer than a less intense but physically longer target. Finally, the interaction of luminance and duration is not significant $[F<1.0)$. This is consistent with the findings by investigators using standard duration discrimination tasks in which the discriminability of the durations of two targets is of focal interest. It has been found in such work that the discriminability of target durations is generally unaffected by the nontemporal information in the two targets (cf. Allan, 1979; Allan \& Kristofferson, 1974b). The lack of interaction among the conditions depicted in Figure 4 suggests that, although the perceived duration of a brief target increases with increasing luminance, the discriminability of that target from another target on the basis of duration is not affected by the luminance level at which the two targets are presented. ${ }^{1}$

It is believed that the results shown in Figure 4 represent a serious challenge to a basic premise of the information-processing models. Perceived duration of a brief target is sensitive to the energy in the target presentation-as affected by either duration or luminance manipulations. This conclusion in turn raises the strong likelihood of contributions from basic sensory processes that have been largely overlooked by existing models of perceived duration. The argument proposed here suggests retinal sluggishness, which alters phenomenal persistence, as the probable candidate for the basis of the luminance effect in this experiment as well as the results in Experiment 1.

\section{GENERAL SUMMARY AND CONCLUSIONS}

This study consists of two experiments that attempted to examine the role of visual persistence effects on a standard temporal judgment task. Specifically, three generally accepted phenomena in the time-perception literature were investigated in terms of sensory persistence differences across stimulus conditions that could account for the results. Experiment 1 compared the effects of stimulus size on both a perceived-duration task and a standard persistence task in which very brief target durations were employed. The striking comparability of results across tasks was discussed in terms of the usable retinal persistence increasing as target size increases. This sensory interpretation was contrasted with the dominant information-processing models favored by other investigators. The so-called empty-filled illusion was also examined in the first experiment by requiring observers to adjust an auditory probe to the phenomenal end of a brief target, which was either an empty field or the same field containing a small, high-contrast stimulus. The results were again consistent with the claim that differences across conditions were due to the varying duration of the contrast-sensitive retinal icon produced by the two types of target. Hence, the results from both phenomena investigated in the first experiment were interpreted in terms of differing degrees of clarity of the fading retinal icons produced by the particular stimulus conditions employed.

One of the important arguments employed by the cognitive processing models against a sensory basis to performance on duration discrimination tasks has been the claimed independence of duration estimates from target luminance effects. The second experiment in the present study examined the effects of target luminance on a standard task of perceived duration. This was undertaken because certain information processing models specifically predict that luminance of a brief target does not alter perceived duration. As predicted from a sensory interpretation of factors underlying task performance, duration estimates were found to increase significantly as target luminance was increased. Therefore, once again the results were entirely consistent with the conclusion that performance on a standard temporal judgment task can be seriously affected by the degree of phenomenal persistence resulting from an energydependent retinal icon.

It is by no means the contention of the present study that all the perceived-duration literature can be reinterpreted in terms of sensory persistence effects. First of all, the durations employed in the present work were extremely brief $(<100 \mathrm{msec})$, because the contribution to performance from retinal persistence would only be predicted for very brief targets of this order of magnitude for which the retina summates the energy in a presentation (i.e., Bloch's law). Many of the studies in the perceived-duration literature characteristically employ stimuli that exceed this level by several orders of magnitude (i.e., seconds). Second, a considerable amount of research in time perception has employed auditory (e.g., Buffardi, 1971; Idson \& Massaro, 1977; Massaro \& Idson, 1976; Thomas \& Brown, 1974) and tactile (e.g., Buffardi, 1971; Craig, 1973) stimulation. The results of the present study do not address these studies. Third, there are numerous findings in the literature that would not seem to depend on sensory effects in any obvious way. Mo and Michalski (1972) demonstrated that the relative frequency of a given stimulus in a block of trials can significantly affect the perceived duration of that stimulus. Avant et al. 
(1975) found stimulus familiarity to influence duration judgments significantly, with nonwords judged longer than words, which in turn were judged longer than letters. And Thomas and Cantor (1975) have reported observers' estimates of target size to increase with increasing target duration. These and numerous other effects reported in the time-perception literature would seem to reflect processes beyond sensory persistence. Nonetheless, there are other findings in the literature that are entirely consistent with the sensory persistence argument proposed here. Several of the masking effects reported by Cantor and Thomas (1976), in which a poststimulus noise mask reduces perceived duration when it quickly succeeds the target, would follow from a prediction of the mask's interfering with fading target persistence. Similarly, the finding by Avant et al. (1975) that increased target contrast significantly increases duration estimate is directly in line with the present argument that stresses clarity of the retinal icon across various stimulus manipulations.

And, finally, the persistence interpretation of a number of temporal phenomena may represent more than an alternate explanation of the specific findings cited above. As Allan (1979) has recently reviewed, there are numerous discrepant studies in the timeperception literature. Frequently, these empirical discrepancies have been attributed to differences in the procedure employed by various investigators. However, the persistence model proposed here stresses the critical role of stimulus variables that can greatly influence retinal persistence. Hence, differences in target size, target luminance, target contrast, target wavelength, retinal location, adaptation level, etc., may well account for the differences across studies by varying the degree of phenomenal persistence that observers may be able to utilize in their judgments. For example, the shape of the psychophysical function relating physical to phenomenal duration may be quite different for high-persistence as opposed to low-persistence conditions. It is known that the persistence from a given target increases as its duration increases (e.g., Long, 1979b; Long \& Sakitt, 1980a, 1980b). Hence, high-persistence conditions (e.g., high-contrast, large, high-luminance targets) will almost certainly exhibit a relationship between physical and phenomenal duration different from that of low-persistence conditions, for which the sensory persistence contribution is minimal. Similarly, results based upon temporal judgments of intervals marked by brief, delimiting flashes may not be directly comparable to those based upon brief stimulus durations, because the role of persistence may be minimal in the former case but marked in the latter. We are currently examining these and other possibilities.

\section{REFERENCES}

Allan, L. G. The perception of time. Perception \& Psychophysics, $1979,26,340-354$.

Allan, L. G., \& Kristofferson, A. G. Judgments about the duration of brief stimuli. Perception \& Psychophysics, 1974, 15, 434-440. (a)

Allan, L. G., \& Kristofferson, A. B. Psychophysical theories of duration discrimination. Perception \& Psychophysics, 1974, 16, 26-34. (b)

Allan, L. G., Kristofferson, A. B., \& Wiens, E. W. Duration discrimination of brief light flashes. Perception \& Psychophysics, 1971, 9, 326-334.

Aluen, F. The persistence of vision. American Journal of Physiological Optics, 1926, 7, 439-457.

Avant, L. L., Lyman, P. J., \& Antes, J. R. Effects of stimulus familiarity upon judged visual duration. Perception \& Psychophysics, 1975, 17, 253-262.

Bowen, R. W., Pola, J., \& Matin, L. Visual persistence: Effects of flash luminance, duration, and energy. Vision Research, 1974, 14, 295-303.

Buffardi, L. Factors affecting the filled-duration illusion in the auditory, tactual, and visual modalities. Perception \& Psychophysics, 1971, 10, 292-294.

Cantor, N. E., \& Thomas, E. A. C. Visual masking effects on duration, size, and form discrimination. Perception \& Psychophysics, 1976, 19, 321-327.

Cantor, N. E., \& Thomas, E. A. C. Control of attention in the processing of temporal and spatial information in complex visual patterns. Journal of Experimental Psychology: Human Perception and Performance, 1977, 3, 243-250.

Coltheart, M. Iconic memory: A reply to Professor Holding. Memory \& Cognition, 1975, 3, 42-48.

Coltheart, M. Iconic memory and visible persistence. Perception \& Psychophysics, 1980, 27, 183-228.

Craig, C. C. A constant error in the perception of brief temporal intervals. Perception \& Psychophysics, 1973, 13, 99-104.

Dick, A. O. Iconic memory and its relation to perceptual processing and other memory mechanisms. Perception \& Psychophysics, 1974, 16, 575-596.

Efron, R. Effect of stimulus duration on perceptual onset and offset latencies. Perception \& Psychophysics, 1970, 8, 231-234.

Eisle R, H. Experiments on subjective duration 1868-1975: A collection of power function exponents. Psychological Bulletin, 1976, 83, 1154-1171.

Eriksen, C. W., \& Collins, J. F. Sensory traces versus the psychological moment in the temporal organization of form. Journal of Experimental Psychology, 1968, 77, 376-380.

Idson, W. L., \& Massaro, D. W. Perceptual processing and experience of auditory duration. Sensory Processes, 1977, 1, 316-337.

Keele, S. W., \& Chase, W. G. Short-term visual storage. Perception \& Psychophysics, 1967, 2, 383-386.

Kristofferson, A. B. A real-time criterion theory of duration discrimination. Perception \& Psychophysics, 1977, 21, 105-117.

LoNG, G. M. Comment on Hawkins and Shulman's Type I and Type II visual persistence. Perception \& Psychophysics, 1979, 26, 412-414. (a)

LoNG, G. M. Iconic memory: Effects of stimulus parameters on short-term visual storage (Doctoral dissertation, Stanford University, 1978). Dissertation Abstracts International, 1979, 39, 4619B. (b)

LoNG, G. M. Iconic memory: A review and critique of the study of short-term visual storage. Psychological Bulletin, in press.

Long, G. M., \& SaKiTT, B. The retinal basis of iconic memory: Eriksen and Collins revisited. American Journal of Psychology, $1980,93,195-206$. (a) 
Long, G. M., \& SakitT, B. Target duration effects on iconic memory: The confounding role of changing stimulus dimensions. Quarterly Journal of Experimental Psychology, 1980, 32, 269-285. (b)

Massaro, D. W., \& Inson, W. L. Temporal course of perceived auditory duration. Perception \& Psychophysics, 1976, 20, 330-352.

Mo, S. S., \& Michalski, V. A. Judgment of temporal duration of area as a function of stimulus configuration. Psychonomic Science, 1972, 27, 97-98.

SakitT, B. Locus of short-term visual storage. Science, 1975, $190,1318-1319$.

Sakitт, B. Iconic memory. Psychological Review, 1976, 83, 257-276.

Sakitт, B., \& Long, G. M. Relative rod and cone contributions in iconic storage. Perception \& Psychophysics, 1978, 23, 527-536.

SAKitT, B., \& Long, G. M. Cones determine subjective offset of a stimulus but rods determine total persistence. Vision $R e$ search, 1979, 19, 1439-1441. (a)

SakitT, B., \& Long, G. M. Spare the rod and spoil the icon. Journal of Experimental Psychology: Human Perception and Performance, 1979, 5, 19-30. (b)

Sperling, G. The information available in brief visual presenta- tions. Psychological Monographs, 1960, 74(11, Whole No. 498). Thomas, E. A. C., \& Brown, I., Jr. Time perception and the filled-duration illusion. Perception \& Psychophysics, 1974, $16,449-458$.

Thomas, E. A. C., \& Cantor, N. E. On the duality of simultaneous time and size perception. Perception \& Psychophysics, $1975,18,44-49$.

Thomas, E. A. C., \& Cantor, N. E. Simultaneous time and size perception. Perception \& Psychophysics, 1976, 19, 353-360.

Thomas, E. A. C., \& Cantor, N. E. Interdependence between the processing of temporal and nontemporal information. In J. Requin (Ed.), Attention \& performance VII. Hillsdale, N.J: Erlbaum, 1978.

Thomas, E. A. C., \& Weaver, W. B. Cognitive processing and time perception. Perception \& Psychophysics, 1975, 17, 363-367.

\section{NOTES}

1. We are indebted to the reviewer for bringing this point to our attention.

(Received for publication March 13, 1980; revision accepted August 26, 1980.) 